\title{
Vaccination of health care workers working in geriatric long term care hospitals reduced patient mortality
}

Potter J, Stott DJ, Roberts MA, et al. Influenza vaccination of health care workers in long-term-care hospitals reduces the mortality of elderly patients. J Infect Dis 1997 Jan;1 75:1-6.

\section{Objective}

To determine whether vaccination of health care workers (HCWs) employed in geriatric medical long term care hospitals and vaccination of long term care patients reduces incidence of influenza and death in patients.

\section{Design}

Randomised controlled trial with 5 month follow up.

\section{Setting}

12 geriatric medical long term care hospitals in Glasgow, Scotland.

\section{Patients}

1059 patients (mean age 77 y, $71 \%$ women) who were residents of a geriatric medical hospital that provided long term care.

\section{Intervention}

Randomisation was done by hospital. 6 hospitals were allocated to a vaccination programme where HCWs were vaccinated, and 6 hospitals were allocated to a no vaccination program. 1078 HCWs were involved in the study. Hospital policies of vaccinating or not vaccinating patients were maintained according to the usual practice. 6 hospitals routinely gave patients vaccinations, and 6 did not.

\section{Main outcome measures}

Patient mortality and occurrence of influenza-like illness.

\section{Main results}

In hospitals where HCWs were offered vaccination, 653 (61\%) were vaccinated. Only 1 patient was vaccinated in a hospital with a policy of not routinely offering patients vaccination compared with $478(89 \%)$ in hospitals where patients were routinely vaccinated. Fewer patients died in hospitals where HCWs were vaccinated than in hospitals where HCWs were not vaccinated $(p<0.01)$ (table), and fewer patients developed suspected viral illness or influenza-like illness $(\mathrm{p}<0.001)^{*}$. No difference was shown in patient mortality in hospitals where patients were offered vaccine and those where patients were not offered vaccine $(p=0.29)$. In a multivariate analysis, non-vaccination of HCWs $(p<0.001)$, a lower Barthel score (functional scale) $(\mathrm{p}=0.004)$, and increased age $(\mathrm{p}=0.03)$ of the patient were associated with increased patient mortality; hospital policy for non-vaccination of patients was not associated with increased mortality $(\mathrm{p}=0.33)$.

\section{Conclusion}

Vaccination of health care workers employed in geriatric long term care facilities was associated with a reduced rate of patient mortality and influenza-like illness.

$* \mathrm{p}$ values calculated from data in article.

Vaccinated HCWs v non-vaccinated HCWs ${ }^{\dagger}$

\begin{tabular}{lllll}
\hline & $\begin{array}{l}\text { Vaccinated } \\
\text { HCWs } \\
\text { EER }\end{array}$ & $\begin{array}{l}\text { Non-vaccinated } \\
\text { HCWs } \\
\text { CER }\end{array}$ & $\begin{array}{l}\text { RRR } \\
(95 \% \text { CI) }\end{array}$ & $\begin{array}{l}\text { ARR } \\
\mid \text { EER - CER } \mid\end{array}$ \\
\hline $\begin{array}{c}\text { Patient } \\
\text { mortality }\end{array}$ & $10 \%$ & $17 \%$ & $\begin{array}{l}41 \% \\
(19 \text { to } 57)\end{array}$ & $7 \%$ \\
\hline
\end{tabular}

${ }^{\dagger}$ Abbreviations defined in Glossary; RRR, ARR, and CI calculated from data in article.

Sources of funding: Greater Glasgow Health Board Care of the Elderly Unit and Medeva.

For article reprint: Professor D J Stott, Academic Section of Geriatric Medicine, Royal Infirmary, Glasgow, G4 OSF, Scotland. Fax +44 (0)1412114944.

Adapted from an abstract published in Evidence-Based Medicine 1997 Jul-Aug;2:121.

\section{Commentary}

This randomised controlled trial by Potter et al is a welcome addition to an important area. It provides evidence of the efficacy of a preventative measure, that is, vaccination of HCWs in long term care hospitals.

The findings of this study showed that patient mortality and rates of influenza were lower when HCWs were vaccinated. It is impressive that $61 \%$ of the HCWs agreed to be vaccinated.

The number of elderly persons in long term care hospitals, and nursing and residential care homes is steadily increasing. Recognising that some elderly persons may not develop adequate antibody con- centrations after influenza vaccination because of their possible impaired immune function, it would seem prudent to offer them protection by vaccinating their HCWs who may transmit the virus.

The findings of this study may have implications for those working in long term care institutions. HCWs are often vaccinated to protect themselves from infections such as hepatitis B. In this instance, however, vaccination can be thought of as a contribution to the protection of elderly patients, many of whom are already at risk because of existing medical conditions which could be further complicated by contracting influ- enza. This study does not indicate whether any side effects were experienced by the HCWs who were vaccinated, therefore one can only assume that there were none to note.

Potter et al conclude that the vaccination of HCWs may also be beneficial to employers because the incidence of influenza will be reduced among staff, and less work time will be lost because of illness.

Veronica E. Johnson-Roffey RGN, RM, RHV Public Health Nurse Coventry Healthcare National Health Services Trust Coventry, UK 